\title{
„Wenn ich die Karrenspur fräße..." - Zur Artikulation von Gefühlen in meineidiger Sprache in einigen Gedichten Paul Celans
}

Artykuł omawia formy artykulacji emocji w niektórych tekstach lirycznych Paula Celana. Emocje są określane jako kody językowe, z którymi teksty Celana wchodzą w dialog. Poetologia sformułowana w Meridianie zakładająca dialogiczną strukturę tekstu lirycznego jako jego otwarcie na Inne niesie ze sobą transcendowanie wszelakich kodów emocji takich jak miłość lub smutek. Szczególnie w obliczu instrumentalizacji języka w socjalizmie narodowym, ale też wypierania holokaustu w czasie powojennym teksty Celana pozycjonują się krytycznie do języka jako systemu reprezentacji. Także możliwość wypowiadania się podmiotu w tekście lirycznym w tekstach Celana jest kwestionowana, ale też broniona.

Der Artikel geht den Artikulationen von Emotionen in einigen Gedichten Paul Celans nach. Emotionen werden als sprachliche und literarische Codierungen aufgefasst, an denen sich die Gedichte abarbeiten. Die im Meridian festgeschriebene dialogische Ausrichtung des Gedichts als Öffnung für das Andere impliziert eine Hinausbewegung über Codierungen von Liebe oder Trauer. Insbesondere angesichts der Instrumentalisierungen der Sprache in der NS-Zeit, aber auch der Verdrängung der Shoah und nicht zuletzt des Verdikts der Unsagbarkeit positionieren sich Celans Gedichte konträr zur Sprache als sprachlichem Repräsentationssystem. Nicht nur die Tragfähigkeit von Zeichenordnungen, sondern der Ort des fühlenden und sprechenden Subjekts steht dabei auf dem Prüfstein.

The article analyzes the articulation of emotions in Paul Celan's poetry. Emotions such as love or grief are shown as language and literary codes each poem has to deal with. Celan's speech The Meridian entails the dialogical structure of poetry as its exposure towards the Other, which understandably implies a critical attitude towards any kind of codification of emotions. Particularly weighty is the historical context: not only the abuse of language during the National Socialism and the suppression of the 
Beate Sommerfeld

Past, but moreover his own doubts related to the ineffability of experiences and emotional states in this singular historical setting make him question the symbolic function of language. Interrogated but shielded is furthermore the position of the lyrical I.

Lyrik gilt als prädestiniert für die Artikulation von Emotionen. Eines der gängigsten Kriterien, mit dem Lyrik von anderen Gattungen abgegrenzt wird, ist das der Subjektivität, die oftmals mit Emotionalität verknüpft wird. Trotzdem zählen die Gefühle in der Dichtung keineswegs zu den favorisierten und systematisch etablierten Forschungsgegenständen. Erst in jüngerer Zeit ist ein zunehmendes Interesse an der Emotionalität von lyrischen Texten zu beobachten, so dass gar von einem „emotional turn“ (vgl. ANZ 2006) gesprochen wird. In dessen Zuge werden die längst überfälligen Fragen sowohl nach der Herkunft als auch Berechtigung der Auffassung von einer besonderen Subjektivität und Emotionalität von lyrischen Texten gestellt (vgl. WINKO 2003:119f). Das Phänomen kann in der Tat durch die literarhistorische Ausprägung der Gattung erklärt werden. Die Auffassung, Lyrik sei die subjektivste und zugleich emotionalste unter den Gattungen, beginnt sich im Zuge der Festschreibung literarischer Gattungsmerkmale im 18. Jahrhundert durchzusetzen. Spätestens seit dem 18. Jahrhundert weisen Gedichte ein besonderes Spektrum an Formen und Inhalten auf, die damit betraut werden, Emotionen zu gestalten und zu vermitteln. Um 1800 werden Subjektivität und Emotionalität der Lyrik bereits systematisch miteinander verbunden, was in Hegels Vorlesungen über die Ästhetik eine kunstphilosophische Untermauerung erfährt. ${ }^{1}$ Hegel zufolge ist „,die innere Subjektivität ... der eigentliche Quell der Lyrik“, diese sei ,auf den Ausdruck rein innerlicher Stimmung, Reflexion u. s.f.“ beschränkt (HEGEL 1970:433). Die sich in dieser Zeit herausbildende Erlebnislyrik kann als ein Prototyp der Gattung gelten. Hegels Ansicht, dass Lyrik wesentlich subjektiv sei, ist weiterhin, über alle methodologischen und ideologischen Differenzen hinweg, der Grundkonsens aller neueren Lyriktheorien, und Gedichte gelten als unmittelbarer Ausdruck eines Subjekts und seiner Stimmungen (vgl. LAMPING 1989:114). Der aus dem Paradigma der Erlebnislyrik hergeleitete Nexus von Lyrik und Emotionalität prägt nach wie vor die Auffassung der Gattung (vgl. LINK 1992:86). Dagegen opponieren die Vertreter der „Modernitätsthese“, dass „die Gestaltung von Emotionen in modernen lyrischen Texten keinen Platz mehr" habe (vgl. WINKO 2003:17). Charakteristisch für diese Position sind Behauptungen wie die Hugo Friedrichs, das moderne Gedicht wende sich als autonomes

Auf Hegel führt auch MeYER-SiCKENDIEK (2001:217) die Gleichsetzung von Lyrik mit Subjektivität und Emotionalität zurück. 
Sprachkunstwerk von dem Anspruch auf Mimesis ab, wolle „nicht dank eines Abbildens von Welt, eines Ausdrückens von Gefühlen“ (FRIEDRICHS 1985: 143) seine Wirkung entfalten, vielmehr zeichne es sich durch Reflexion aus, sei ein „kühles Operieren“ mit dem Sprachmaterial (FRIEDRICHS 1985:29). Trotz Abgrenzungsversuche, führt DIETER LAMPINGs (1991:10) Ansicht, dass moderne Lyrik einen Bruch mit der Erlebnislyrik vollziehe (LAMPING 1991: 10) und ,traditionelle Kategorien wie ,Stimmung“, ,Erlebnis“ oder ,Innerlichkeit“" vor dem Phänomen der modernen Lyrik offenbar ,versagen“ (LAMPING 1989:135), in die gleiche Richtung.

Um die Emotionalität von lyrischen Texten nicht voreilig gegen deren Reflexivität auszuspielen, scheint es zunächst nötig, den Emotionsbegriff zu klären - zum einen, um verbreitete Auffassungen nicht unreflektiert zu repetieren, und zum anderen, um die Anschlussmöglichkeiten zwischen literaturwissenschaftlichen Perspektiven und der multidisziplinären Emotionsforschung zu erkunden. Eine Operationalisierung des Emotionsbegriffs erscheint besonders angesichts der unklaren Forschungslage innerhalb der Emotionsforschung selbst angeraten, in der allenthalben die Konturlosigkeit des Gegenstandsgebietes Emotionen beklagt wird. ${ }^{2}$ Eine erste zu treffende Unterscheidung ist die zwischen Gefühl und textualer Repräsentanz. Gerade der ,emotional turn ‘ in den Sprach- und Literaturwissenschaften eröffnet eine neue analytische Perspektivierung, die die Medialität der psychischen Realität erlebter Emotionen in den Fokus rückt. So betrachtet NORBERT FRIES (2007:293-337) Emotionen als spezifische, durch Zeichen codierte Bedeutungen, und etabliert systematische Form-Bedeutungs-Beziehungen. In der systemtheoretischen Sichtweise NiKLAS LuHManNs (1982:31) wird der Terminus ,Codierung' auf die Ausbildung von Leitdifferenzen begrenzt, die die gesellschaftliche Kommunikation strukturieren und steuern. Luhmann verwendet den Begriff in erster Linie, um das Phänomen Liebe als Medium gesellschaftlicher Kommunikation zu analysieren. Anschlussfähiger scheint die systemtheoretische Sichtweise von HEINZ-GÜNTER VESTER (1991:74), nach dem Emotionen insofern als codiert betrachtet werden, als sie über prototypische „Skripts“ oder Szenen vermittelt werden. Emotionen sind demnach textlich codiert, werden textuell gespeichert und kulturell tradiert. Codes werden als sprachliche, gestische und mimische Muster aufgefasst, die in einer Kultur als Gefühle evozierend oder vermittelnd gelten. Für die Analyse der Emotionalität literarischer Texte stellt SABINE WINKO (2003:85-90) eine Auffassung von

2 Vgl. den Überblick bei BöHME (1997:525-548). 
Emotionen als textuellen Phänomenen zur Disposition. Insgesamt setzt sich die Auffassung durch, dass Darstellungen von Emotionen in der Literatur nicht ausdruckspsychologisch, sondern grundsätzlich nur als symbolische Verfahren zu beschreiben sind (vgl. beispielsweise KocH 2007:48). Fokussiert werden müssen die ästhetischen Strategien, durch die das NichtSichtbare der Emotionen zur Erscheinung zu bringen (vgl. ISER 1991:504f.) und in einem symbolischen Zeichensystem verfügbar zu machen ist. Damit aber bewegt sich die Darstellung von Emotionen in literarischen Texten in einem Spannungsfeld von Affekthaftigkeit und symbolischer Kommunikation. ${ }^{3}$

Celans Gedichte positionieren sich gegenüber den in der lyrischen Tradition herausgebildeten Codierungen und arbeiten sich an ihnen ab. Wo sie Gefühle artikulieren wollen, stehen sie in einem Spannungsverhältnis zu den vorgefundenen Emotionscodes. In Anschlag zu bringen sind die poetologischen Aussagen im Meridian, seiner Rede zur Verleihung des Büchner-Preises durch die Deutsche Akademie für Sprache und Dichtung in Darmstadt, der als einem der wenigen poetologischen Manifeste nach 1945 besondere Beachtung zukommt (vgl. KORTE 1989:91). Dichtung soll dem ganz persönlichen Dasein des Individuums zum Ausdruck verhelfen, ihr wird von Celan eine existentielle Dimension zugeschrieben. Als Vorbild gilt ihm Georg Büchner, Muster eines Dichters, der dichterisches Sprechen als Artikulationen eines unverwechselbaren Individuums realisiere und „unter dem Neigungswinkel seines Daseins, seiner Kreatürlichkeit“ (CELAN 1983b:197) schreibe. ${ }^{4}$ Damit distanziert sich Celan von der Auffassung des Gedichts als eines autonomen Sprachkunstwerks. Das absolute und monologische Gedicht wird dem „Medusenhaupt“ einer unmenschlichen Kunst zugeschlagen, die den Selbstausdruck verhindere und „Ich-Ferne“ (CELAN 1983b:193) schaffe. Celans Gedichte wollen nicht absolut und monologisch sein, sondern sind in eine dialogische Konstellation eingebunden als Öffnung des Textes auf ein

3 Wirkungsästhetische Ansätze sollen außer Acht gelassen werden. Der Ansatz von ANZ (2011:113-129) beispielsweise, der die in literarischen Texten gestalteten Emotionen wieder auf „,natürliche“ Situationen rückbezieht (der Leser könne anhand literarischer Texte Emotionen spielerisch erproben), simplifiziert die Komplexität der Zusammenhänge zwischen Emotion und literarischem Text.

$4 \quad$ Vgl. auch die Forderung aus der Bremer Rede: Im Gedicht sei nicht die Sprache als solche am Werk, ,sondern immer nur ein unter dem besonderen Neigungswinkel seiner Existenz sprechendes Ich, dem es um Konturierung und Orientierung geht." (CELAN 1983b:168) 
Gegenüber: „Das Gedicht will zu einem Anderen, es braucht dieses Andere, es braucht ein Gegenüber. Es sucht es auf, es spricht sich ihm zu." (CELAN 1983b:198) Jedes Gedicht - Celan spricht nicht von der Lyrik als Gattung, sondern stets vom Gedicht als konkreter sprachlicher Konkretisierung - steuere auf ein „Geheimnis der Begegnung“ (CELAN 1983b:198) zu. Der dialogische Charakter des Gedichts meint die Möglichkeit der Begegnung mit dem Anderen: „Vielleicht - ich frage nur -, vielleicht geht die Dichtung, wie die Kunst, mit einem selbstvergessenen Ich zu jenem Unheimlichen und Fremden, und setzt sich - doch wo? Doch an welchem Ort? Doch als was ? - wieder frei.“ (CELAN 1983b:193) Das Du in Celans Texten ist immer „eine Gestalt des Anderen, im Zuhalten auf dieses Du öffnet das Ich sich, wird exzentriert und gewinnt selbst Gestalt" (BÖNING 2005:228). Dieses befreiende Sich-Öffnen für das Andere impliziert eine Hinausbewegung über die Symbolisierungen der Sprache, meint insbesondere auch eine Befreiung der Sprache aus den fatalen Instrumentalisierungen während der NS-Zeit und den Verdrängungen der nazistischen Vergangenheit in der Nachkriegszeit. Die angetroffene Sprache wird für Celan zur meineidigen Sprache, in der die Erfahrung der Shoah entweder gar nicht oder falsch repräsentiert ist. So meint das Sprechen von Ich und Du im Gedicht oftmals, einander „Dunkles“ zu sagen (CELAN 1983:37), aus den symbolischen Repräsentationspraktiken Ausgeschlossenes, in eigenen Traumatisierungen Verkapseltes und mit dem Verdikt der „Unsagbarkeit“5 Belegtes. Dieses Textbegehren, dem Anderen zum Ausdruck zu verhelfen, steht damit konträr zu den sprachlichen Codierungen. Im Gedicht soll die Sprache befreit werden und gleichsam neu auferstehen, aus ,tausend Finsternissen todbringender Rede wieder zutage treten“" (CELAN 1983b:186), wie es in Celans Bremer Rede heißt. Wie dieser Dialog Celans mit den sprachlichen Überformungen von Emotionen vonstatten geht, um das Gedicht auf den ganz eigenen Weg zum Fremden, Anderen zu schicken, soll im Folgenden an einigen Textbeispielen nachvollzogen werden.

\section{„Fühlwörter“ am Abschiedsgrat}

Das Gefühl, das bei weitem am häufigsten in der Literatur zur Sprache kommt und von ihr in verschiedenen Varianten gebildet, codiert und geprägt wird, ist die Liebe. Stets als einzigartig und individuell erlebt, ist sie das am ausgiebigsten kultivierte und literarisierte Gefühl. Da es kaum einen Satz

5 Zu Celans Auseinandersetzung mit Adorno vgl. KIESEL (2004:453f.). 
über Liebe gibt, der nicht bereits in einem Liebesgedicht formuliert wurde, situiert sich lyrisches Sprechen über Liebe in ganz besonderer Weise im Spannungsfeld von subjektivem Gefühl und symbolischer Repräsentation. Den Codierungen der Liebe geht LUHMANN (1982) aus systemtheoretischer Perspektive nach. Der Strukturalist Roland Barthes weist in seinen Fragmenten einer Sprache der Liebe nach, dass das Sprechen über Liebe sich nur in Floskeln vollzieht, die sich zu einer uralten und ahistorischen Sprachmaschine des Banalen fügen. Das Ich in der Sprache der Liebe sei stets eine Montage, zu seiner Konstitution sind Bruchstücke verschiedensten Ursprungs montiert worden, die kulturgeschichtlichen und kanonisierten literarischen Texten entstammen (vgl. BARTHES 1977:21). Dass der Liebende keine andere Wahl hat, als sich dieses Liebesdiskurses zu bedienen, macht Barthes in seinem Text sinnfällig, indem er dem Diskurs immanent bleibt und verschiedene Sprachszenen reflektierend nachvollzieht. Celans Gedichte arbeiten sich an Emotionscodes ab, wenn in seinen Gedichten Liebe gestaltet wird, wobei sich Celan innerhalb der Codierungen von Liebe bewegt und sie als Vehikel der Artikulation von Gefühlen benutzt. So evoziert das Gedicht Fernen aus dem Gisèle Lestrange gewidmeten Band Sieben Rosen später eine typische Liebesszene: „Aug in Aug, in der Kühle“ um zur Aufforderung überzuleiten: „laß uns auch solches beginnen“ (CELAN 1983:95). Bis in die späten Gedichte konstruieren Celans Texte immer wieder Beziehungsszenen, die auf der Folie eines Liebesgedichts zu lesen sind und den Liebescode reflektierend nachbuchstabieren, wie im folgenden Text aus dem Band Lichtzwang von 1970: „Ich kann Dich noch sehn: ein Echo,/ ertastbar mit Fühl-/ wörtern, am Abschieds-/grat.// Dein Gesicht scheut leise,/ wenn es auf einmal/ lampenhaft hell wird/ in mir, an der Stelle,/ wo man am schmerzlichsten Nie sagt.“ (CELAN 1983a:275)

Der Abschied von der Geliebten ist ein soziales Setting, eine Beziehungsszene. Das dialogische Strukturprinzip schafft den Raum zur Ich-Artikulation: „Das Ich konstituiert sich am Du, die Ich-Rede ist Anrede.“ (RIEDEL 1989:29) Ohne ein imaginiertes Gegenüber scheint das lyrische Sprechen nicht tragfähig, flaut seine Dynamik ab. Aber die Aufrechterhaltung des Beziehungsgefühls ist prekär, ist vom Zeitverlauf und räumlicher Entfernung bedroht. Das Gedicht rekurriert auf eine Verlusterfahrung, die negativ besetzt ist, nimmt aber auch Bezug auf die Codierungen der Liebe, in die die Abwesenheit schon eingeschrieben ist. So weist Barthes der Abwesenheit des geliebten Menschen einen festen Platz im Liebesdiskurs zu: Liebe speist sich aus Abwesenheit (vgl. BARTHES 1977:185), das Negative wird mitgenossen und dient dazu, die Liebe bewusst zu machen und wachzuhalten (vgl. LuH- 
MANN 1982:83). In Celans Text konstituiert sich die Bezogenheit auf das Du als Bezug auf ein Abwesendes, nie einzuholendes Gegenüber. Das Gedicht agiert dabei in besonderer Weise die Opposition von Anwesenheit und Abwesenheit aus: Es wird zunächst eine Abwesenheit konstruiert, um die Möglichkeiten ihrer Überbrückung durchzuspielen. Diese liegen in der sprachlichen Repräsentation des Gegenüber: Das Du ist gerade noch erreichbar von einer Sprache, die mit „Fühlwörtern“ operiert. Wörter ersetzen die reale Präsenz des Objekts, in ihnen kann sich - unabhängig von der realen Existenz des Anderen - ein Beziehungsgefühl einnisten. Celans Text hält damit die Balance zwischen Anwesenheit und Abwesenheit. Die Wörter der Poesie werden zu Statthaltern der sensuellen Erfahrungen, der Fernsinne Sehen, Hören, Riechen, welche die Objekte näher rücken lassen und ertastbar machen. Das im Gedicht etablierte Beziehungsgefüge bezieht also die Sprache mit ein. Das Gelingen der sprachlichen Repräsentation, das Sprechen mit „Fühlwörtern“ wird als beglückend, aber auch als prekär erlebt. Sprache und Gefühle sind gerade noch zur Deckung zu bringen, sind eine Gratwanderung mit Worten. Denn: „Worte trennen stärker als Körper“ (LuHMANN 1982:89). Das Gedicht ist in einem Gerade noch angesiedelt, das sich nicht nur auf das Entfernen der Geliebten beim Abschied bezieht, sondern das Problem der sprachlichen Repräsentierbarkeit von Gefühlen exponiert. Durch die sprachlichen Inszenierungen wird im Gedicht die Anwesenheit einer Abwesenheit entworfen. Die physische Anwesenheit der Geliebten und ihre symbolische Präsenz sind in Celans Text nur als Alternative denkbar: Die Abwesenheit der Geliebten ist somit die Voraussetzung für ihre symbolische Anwesenheit: Zunächst muss der geliebte Mensch aus dem Text „verabschiedet“ werden, um das Gefühl zu ihm symbolisch verfügbar zu machen. Erst dieses Aufgehoben-Sein, die Präsenz des Gefühls in der Sprache sichert sein Fortbestehen. JACQUES LACAN (1978:166) fasst dies in dem gewichtigen Satz zusammen: „Das Symbol stellt sich so zunächst als Mord der Sache dar, und dieser Tod konstituiert im Subjekt die Verewigung seines Begehrens.“

Gefühle und Sprache stehen damit in einer konfliktreichen Beziehung zueinander. Das authentische Gefühl, das von der Sprache nur verfälscht werden kann, ist einer der Topoi der Empfindsamkeit in der zweiten Hälfte des 18. Jahrhunderts. Einerseits scheint die Sprache zu versagen, wenn es darum geht, die Flüchtigkeit und zugleich Intensität von Gefühlen auszudrücken, andererseits ist sie die symbolische Form, die gerade die Artikulierung von Gefühlsszenen und Gefühlsdramen ermöglicht. Die Sprache mit ihren symbolischen Codierungen erst lässt aus physischen Bedürfnissen und inkommensurablen Triebschüben die differenzierteren Empfindungen als Gefühle 
erwachsen. Indem sie als symbolisches Medium Gefühle überhaupt erst erfahrbar macht, schafft sie aus einem labilen und undifferenzierten Gemenge von Affekten die Gefühle - ein Paradox, das Friedrich Schiller in den Distichen der Xenien pointiert: Im Distichon Sprache beklagt er, dass der tote Buchstabe und die Seele, traditionell der Ort der Gefühle, nicht zusammenkommen können: „Warum kann der lebendige Geist dem Geist nicht erscheinen!/ Spricht die Seele, so spricht, ach! schon die Seele nicht mehr." Im darauffolgenden unter dem Titel An den Dichter wird diese Unvereinbarkeit in das Bild der Liebenden und des Liebesakts gefasst, als Körper trennt die Sprache und stellt zugleich das einzige Medium der Vereinigung dar: „Laß die Sprache dir sein, was der Körper den Liebenden; er nur/ ists, der die Wesen trennt und der die Wesen vereint.“ (SCHILLER 1943:302) Ähnlich wie die Liebe, der die so sehnsuchtsvoll erwartete Verschmelzung der Körper nicht genügen kann, muss das Begehren des Schreibenden, das Gefühlte in Worte zu fassen, unstillbar bleiben. Wie die Opazität des Körpers des geliebten Menschen ist die Sprache ihm im Weg, zugleich aber stellt sie den einzigen Weg, das einzige Mittel dar, das ihm zur Verfügung steht, um das stets sich Entziehende zu fassen. Unendliche Annäherung, nie zu überbrückende Trennung, unstillbares Begehren - diese Paradoxien beschreiben die Liebe ebenso wie die sprachlichen Artikulationsversuche des Dichters. Liebe ist ein Gefühl, das einer Ökonomie der Verausgabung, des Verströmens folgt (vgl. BARTHES 1977:99). Ihr ist die Struktur eines endlosen Begehrens nach dem Abwesenden eigen (BARTHES 1977:185f.), das ihr zu ihrer eigenen Verstärkung dient und in der sprachlichen Repräsentation arretiert wird. Das „Nie“ am Ende von Celans Gedicht ist demnach nicht nur auf die Codierung von Liebe als eines unerfüllten Gefühls (LUHMANN 1982:75) zu beziehen, sondern ebenso auf das unstillbare Begehren sprachlicher Repräsentierung von Gefühlen.

Eine rein abstrakte Verhandlung der Abwesenheit im Sinne sprachlicher Nichtrepräsentierbarkeit würde jedoch die existentielle Dimension der Gedichte verfehlen. Die Erkenntnis einer nie einzuholenden Absenz, von der Celans Gedichte durchdrungen sind, speist sich vor allem aus der Erfahrung der Shoah. Das Gedächtnis der Toten ist es, die sich als Verlusterfahrung in die Texte einschreibt, und diese Trauer über die unwiderrufliche Abwesenheit durchdringt auch die Liebe. Die in Celans Texten zum Ausdruck drängenden Emotionen, allen vorweg die Liebe, sind damit todverschwistert ${ }^{6}$ :

6 Vgl. Stellen wie: „Am lichtesten brannte das Haar meiner Geliebten:/ ihr schick ich den Sarg aus dem leichtesten Holz“ (CELAN 1983:31). 
Lieben ist nur „,von Rauchmund zu Rauchmund“ (CELAN 1983a:59) denkbar. Selbst noch im Evozieren der Liebe als eine Gegenkraft gegen die etablierte Ordnung von Henkern und Opfern ${ }^{7}$ bleibt jedem Gedicht sein Datum, sein „20. Jänner“ (CELAN 1983b:196) ${ }^{8}$ eingeschrieben. Wenngleich die Emotion Liebe in Celans Texten überaus präsent ist, sollte man daher sich nicht zu einer verkürzenden Leseweise seiner Gedichte als ,Liebesgedichte verführen lassen. Besonders die Texte, die in die Zeit seiner Liebesbeziehung zu Ingeborg Bachmann fallen, verleiten zu der billigen These eines Dialogs in Liebesgedichten, die sich in Teilen der Forschung breitgemacht hat und die sicherlich mit Vorsicht zu genießen ist. ${ }^{9}$ Gerade bei Texten wie Corona, in dem Celan die Beziehung zu Bachmann aufarbeitet, wird der Holocaustbezug gerne gegen eine - vielleicht für den Leser gefälligere - Lektüremöglichkeit als Bachmann gewidmetes Liebesgedicht ausgespielt. In Celans Texten wird beides jedoch zusammengedacht - Celans Begegnungen mit einem Du stehen damit unter dem Zeichen des „20. Jänner“, das Artikulieren von Emotionen ist ohne den dialogischen Bezug auf die verdrängte/vergessene Vergangenheit nicht denkbar: Lieben kann man einander nur ,wie Mohn und Gedächtnis“(CELAN 1983:37).

\section{Sprechen am Ort einer Abwesenheit - Artikulationsformen der Trauer}

Auch in den Trauertexten Celans wird die Grenze zwischen An- und Abwesenheit ausgehandelt. Wie in der Liebe konstituiert sich das sprechende Ich in der Erfahrung des Mangels, der Abwesenheit des Anderen. Trauer bezieht sich auf einen verlorenen Anderen, um dessen Abwesenheit sie als um ihr leeres Zentrum kreist. Die in Texten artikulierte Trauer bildet damit eine Grundfigur einer Sprache des Mangels, kann als Paradigma eines Sprechens am Ort einer unerträglichen Abwesenheit gelten. Das Ich, sein Bewusstsein und seine Sprache formieren sich, wie JACQUES DERRIDA (1988:57) schreibt, in der Einsamkeit, im Begehren nach dem verlorenen Anderen: „Es ist diese

7 Vgl. beispielsweise: „Im Quell deiner Augen/ erwürgt ein Gehenkter den Strang.“ (CELAN 1983:33)

8 Im Meridian ist der 20. Jänner, der Tag, an dem Lenz in Büchners Lenz „,durchs Gebirg“" ging, aber für Celan zuerst der Tag der Wannseekonferenz 1942, die die Vernichtung der Juden beschloss.

9 Vgl. die kritische Erörterung in WeIGEL / BöSCHENSTEIN (1997:9f.). 
schreckliche Einsamkeit, die meinige oder die unsrige, beim Tode des anderen, die jene Selbstbeziehung konstituiert, die man ,ich', ,uns ', [...], Subjektivität', ,Gedächtnis‘ heißt.“

Celans Trauertexte werden in den Widerspruch von Gefühl und Sprache hineingezogen. Was auch immer gesagt wird, muss sich am Verdikt des Unsagbaren (Adorno) messen, ist also Sprechen aus einer Negativität heraus, das sich seiner prekären Lage stets bewusst ist und daher stets auf sich selbst reflektiert. Das Ausschließungsverhältnis von Trauer und Sprache befördert eine Negativität, angesichts deren sich jede Rede der Trauer als ein Sprechen „trotzdem“ positioniert. Auch die Artikulation von Trauer im Gedicht bewegt sich damit in der Spannung von Affekthaftigkeit und symbolischer Kommunikation. Wie Liebe ist Trauer ein individuell empfundenes, oft überwältigendes Gefühl, das sich verausgaben will, und sich nicht durch die Codierungen der Trauer befrieden lässt. Jeder Trauertext führt daher eine Auseinandersetzung mit kulturellen Überformungen und Darstellungskonventionen von Trauer. Indem die eingespielten Bilder und Gesten der Trauer und Totenklage als ein Arsenal von Stoffen wirksam wird, das sich wie ein Schutzschirm vor die eigene unsagbare und schmerzende Leere schiebt, verhindern sie Alteritätserfahrungen. Dies steht gerade bei Celan dem Postulat einer Dichtung entgegen, die der Begegnung mit dem Anderen dienen soll. In seinen Texten sucht sich die Trauer Artikulationsmöglichkeiten abseits der Codierungen. Im Aufbrechen der Codierungen soll Trauer entritualisiert und der individuellen Erfahrung verfügbar gemacht werden. Sie wird ihres kulturellen Habitus entkleidet als etwas, dem nur die private Auseinandersetzung des Trauernden mit dem Verlorenen gerecht werden kann. So individualisiert, ist Trauer allerdings kaum mehr kommunizierbar und gerät in das Register des Inkommensurablen, wird ein ,unsagbares“ Gefühl ${ }^{10}$, dem das Register sprachlich-rhetorischer Codierung nicht mehr genügen kann. Das Ich spricht aus einer Position der Unmöglichkeit, lyrisches Sprechen wird zur unlösbaren Aufgabe: zu sprechen, wo das Gebot der Singularität jede Mitteilbarkeit obszön zu machen scheint. Das Problem soll am Gedicht Eine Gauner- und Ganovenweise gesungen zu Paris emprès Pontoise von Paul Celan aus Czernowitz bei Sadagora aus der Niemandsrose illustriert werden. Celan bedient sich hier der Folie des Liedes, das prädestiniert scheint zum Ausdruck von Emotionen. Celans „Lieder“ bezeichnen allerdings eher die kontradiktorische Wiederaufnahme der Gattungsvorgaben. Wir haben es mit

10 Zur Entfaltung des Unsagbarkeitstopos der Trauer im 18. Jahrhundert vgl. KoCH (2007:147f.). 
einem intellektuellen Aufbrechen der romantischen Liedform zu tun, wie sie bereits Heinrich Heine im Buch der Lieder vorführt, der im Untertitel des Textes zitiert wird. Mit der Wahl der intertextuellen Folie der „Gaunerweise“ legt das lyrische Ich sich zugleich eine Identität zu: die des Bänkelsängers. Es ist das einzige Gedicht, in das Celan sich selbst mit Namen einbringt, was nicht autobiographisch zu verstehen ist, sondern ein spielerisches Verfahren darstellt, das sich aus der Wahl der Textform ergibt. ${ }^{11}$ Wenn sich der DichterAutor in seinem Text selbst nennt, ist dies Teil einer Selbststilisierung: Der Autor tritt uns in der Rolle eines Bänkelsängers entgegen. Was hier aber letztendlich auf dem Prüfstein steht, ist der Ort des sprechenden Subjekts: Das Ich geht nur in intertextueller Vermittlung und damit in reflexiver Brechung in den Text ein. Damit wird einer subjektzentrierten Zeichenpraxis eine Absage erteilt, die sich als unvermittelte Sprache der Seele gebärdet, dabei aber ein Weg gefunden, das Ich im Text präsent zu halten. Das Ich ist nur aus der von der Kunst erzeugten „Ich-Ferne“ heraus im Gedicht zugegen. Im intertextuellen Spiel mit der Folie der „Gaunerweise“ und dem ruppigen Ton der marodierenden Soldateska wird eine Strategie der Selbstdistanzierung verfolgt, die der Distanzierung von den eigenen, schmerzhaften Emotionen dient. Im Zentrum des Textes steht die Trauer um den Dichterfreund Ossip Mandelstam. Der Lyriker Mandelstam erlangt als vom totalitären System Verfolgter, Dichter und Jude für Celan gleichsam brüderliche Qualitäten (vgl. BÖSCHENSTEIN 1988:155-168). Es ist naheliegend, eine assoziative Verknüpfung des Namens Mandelstam mit dem Wort „Mandelbaum“ zu vermuten (vgl. NAJDITSCH 1998:687f.), zumal der Band Niemandsrose dem Dichter „Mandelstamm“ gewidmet ist und durch die Schreibweise - Celan schrieb den Namen durchgängig mit einem doppelten „m“ - der Name des Dichters eine Metaphorisierung erfährt und mit dem „Mandelbaum“ der Gaunerweise kontaminiert wird. Allerdings sind die Verweise des Motivkomplexes Mandel und Mandelbaum in Celans Euvre so zahlreich, dass über den persönlichen Bezug hinaus eine Bezugnahme auf die Welt des Judentums angenommen werden kann, die als Gegenwelt zur existierenden ins Feld geführt wird und auf deren Untergang das Subjekt mit tiefer Trauer reagiert. Der „Mandelbaum“ wird in Celans Text somit zu einem Zeichen der Abwesenheit.

Ein Blick in die Traumaforschung eröffnet ergänzende Bezüge. Der Mandelkern (Amygdala) ist der Teil des Gehirns, in dem die Eindrücke zunächst

11 Einen detaillierten Nachweis über die intertextuellen Bezüge führt SCHULZ (2002:108f.). 
aufgenommen werden, um sie einer Prüfung zu unterziehen, eine Art Gefahrenmeldestelle. Erst wenn das Ereignis als emotional nicht übermäßig belastend befunden wird, werden die Erinnerungen daran in den Hippocampus des Temporalhirnlappens überführt und gelangen damit in das explizite, hippocampale Gedächtnis. Erst dort angelangt, kann das entsprechende Ereignis narrativ biographisch, d.h. zeitlich chronologisch und sprachlich ausdrückbar erinnert werden (vgl. HUBER 2007:44-51). Angesichts der Tatsache, dass Celan mit den Grundlagen der klinischen Neurobiologie recht gut vertraut war und häufig seine poetologischen Aussagen in das Gewand neurobiologischer Terminologie kleidet, erscheinen solche Bezüge immerhin möglich. Das Gedicht wäre in dieser Lesart der Ort, an dem vorgeführt werden kann, wie die Überführung ins hippocampale Gedächtnis aufgrund der vom „Mandelkern“ als übermäßig befundenen Belastung durch das zu Erinnernde nicht vollzogen werden kann. Folge ist die Unfähigkeit zu narrativer, chronologischer und sprachlicher Artikulierung des traumatisierenden Ereignisses. Die Erinnerung kommt gleichsam an der Gefahrenmeldestelle nicht vorbei und umkreist den „Mandelkern“. Wir hätten es demnach hier nicht mit einem metaphorischen Bezug zu tun, sondern mit dem Versuch, neurobiologische Prozesse mit den Mitteln der Literatur nachzubilden. Im achtsamen Umkreisen der Worte Mandelstam bzw. Mandelbaum wird das Verfahren der Affektregulierung performativ realisiert. In Offene Glottis ist die Rede vom „Reizschutz Bewußtsein“ (CELAN 1983a:388), eine Formulierung, die im ersten Entwurf zum Gedicht erhellt wird, wo sich unter der Überschrift „Benjamin“ die Notiz: „Bewußtsein als Reizschutz - Freud“ findet (CELAN 1994:158). In seinem Exemplar von Jenseits des Lustprinzips strich Celan den Satz an: „Wir haben bisher ausgeführt, daß das lebende Bläschen mit einem Reizschutz gegen die Außenwelt ausgestattet ist." (CELAN / CELAN-LESTRANGE 2001:296) ${ }^{12}$ Folgt man Freuds Auffassung, so dient das Bewusstsein dazu, dem Menschen die wirklichen Eindrücke der Welt vorzuenthalten. Mehr als Stichproben der Außenwelt macht es ihm um seiner Unversehrtheit willen nicht zugänglich (vgl. BÖNING 2005:229). Damit vermittelt das Bewusstsein nicht die Wahrheit von der Welt, ausgenommen sind die traumatischen Erfahrungen, die den Reizschutz des Bewusstseins außer Kraft setzen (vgl. BÖNING 2005:232). Diese Schutzfunktion des Bewusstseins wird von Celan mimetisch nachgebildet und an ihre Grenzen geführt, womit die Texte das Gegeneinander von Bewusstsein und Trauma ausagieren.

12 Den Hinweis verdanke ich BÖNING (2005:229). 
Die Schwierigkeit der Trauer, zur Sprache gebracht zu werden, wird damit im Text performativ zur Geltung gebracht. Der Inhalt wird durch rhythmisierte Sprechgesänge, wortspielerische Verhunzungen und Schüttelreime quasi aus dem Text herausgeschrieben, bis am Ende etwas kaum noch Erkennbares übrigbleibt: „Denn es blühte der Mandelbaum./Mandelbaum, Bandelmaum.// Mandeltraum, Trandelmaum./ Chandelbaum.// Heia./ Aum.“ (CELAN 1983:229) Die Interjektion „Heia“ treibt nur noch den Wortstumpf „Aum“ aus sich hervor, der keinerlei Bedeutung mehr vermittelt und die Perspektive endgültigen Schweigens auftut. Die Verwandlungen des „Mandelbaums" sind als Artikulationsformen der Distanzierung zu lesen, durch das Verfahren des melancholischen Sprachspiels, das Gefühle zu inhaltslosen Regungen werden lässt, wird die Überwältigung durch Trauer aufgefangen. Sowohl der „Mandeltraum“, in dem noch einmal ein Moment der Positivität anklingt, als auch der „Bandelmaum“ mit den Erinnerungen an Strick und Galgen sind im Raum des Kunstwerks aufgehoben. Immer wieder wird die Intensität der negativen Emotionen gemildert, und das Gedicht wird zum emotionalen Schutzraum, in dem sich das Ich in selbstbemessenen Dosierungen seinen Traumatisierungen zu stellen vermag. Die Folie des Bänkelsangs wird als rhythmische Vorgabe wirksam, die die Dynamik des Textes in Gang hält. Die semantischen Valenzen werden durch Rhythmisierung überlagert, ein Mechanismus der Selbstaffektion durch Rhythmus, Bewegung und Stimme kommt zum Tragen, der der Affektentladung dient und die Versprachlichung von Emotionen in der emotionalen Grenzsituation der Trauer zulässt.

Durch Sprechgesänge, Kehrreime wird eine hypnotische Wirkung erzielt und die Selbstvergessenheit angestrebt, die Celan im Meridian der Kunst zuschreibt: „Denn Kunst schafft Ich-Ferne“ (CELAN 1983b:193). Büchners Lenz wird von Celan poetologisch gedeutet: In diesem Zustand der Selbstvergessenheit wird das Ich für die Erfahrung des Anderen geöffnet: „Vielleicht ich frage nur -, vielleicht geht die Dichtung, wie die Kunst, mit einem selbstvergessenen Ich zu jenem Unheimlichen und Fremden, und setzt sich - doch wo? Doch an welchem Ort? Doch als was? - wieder frei." (CELAN 1983b:193) Nur aus der „Ich-Ferne“ heraus ist das Ich imstande, sich seinem Schmerz zu stellen. Das Gedicht ermöglicht ein Absehen von sich selbst, schafft einen emotionalen Schutzraum, in dem Traumen gelöst und Emotionen freigesetzt werden. Es wird eine Gleichzeitigkeit von Selbstvergessenheit und Eingedenken geschaffen, die von Celan in die Formel „Mohn und Gedächtnis“ (CELAN 1983:37) gebracht wird. So wird die dialogische Dimension des Gedichts virulent, kann das Gedicht die Begegnung mit dem Anderen, 
der Alterität des Todes, herbeiführen, im Zuge deren Traumatisierungen bezwungen werden und eine Trauerarbeit initiiert wird, die sich nicht in der symbolischen Repräsentation fixieren lässt. Innere Betroffenheit und textuale Darstellung greifen abseits der Codierungen von Trauer ineinander. Trauer wird nicht einfach im Text abgebildet, der Text macht sich vielmehr zur Bühne eines Vollzugs. In den rhythmischen Sprechgesängen wird die Abundanz der Trauer nachgebildet, die sich verströmen will. Das „Zerschreiben“ des Namens ist als Strategie der Verweigerung von Referenz ${ }^{13}$ lesbar, die einer Logik des Ersetzens des Verlorenen durch Zeichen folgt. Celans Gedichte wollen damit einer symbolischen Aneignung des Totengedenkens entgehen, die die Abwesenheit der Toten und die Alterität des Todes verneint. Die Verweigerung von Repräsentation steht für das Aushalten von Abwesenheit und Fremdheit, das an den Punkt der Umkehr und zur Begegnung mit dem Anderen führt:

Dichtung: das kann eine Atemwende bedeuten. Wer weiß, vielleicht legt die Dichtung den Weg - auch den Weg der Kunst - um einer solchen Atemwende zurück? [...] vielleicht gelingt es ihr hier, zwischen Fremd und Fremd zu unterscheiden. [...] Vielleicht wird hier, mit dem Ich - mit dem hier und solcherart freigesetzten befremdeten Ich, vielleicht wird hier ein Anderes frei? (CELAN 1983:195f.)

Dabei soll die symbolische Ordnung, die in Auschwitz falsifiziert wurde, zerbrochen werden. Die Destruierung der diskreditierten Sprache vollzieht sich im Unterlaufen der Erwartung sinnhafter, quasi natürlicher Rede, im Ausscheiden aus dem Bereich des Logos, des Sinns, so wie es Lenz bei Büchner widerfährt. Letztendlich aber kann dies nur auf Kosten des Verlusts an Bedeutung erreicht werden - es erweist sich, dass Trauer sich nicht in der Sprache, sondern in deren Ausfall artikuliert. Nur so, nur wenn sie durch „ein furchtbares Verstummen“ (CELAN 1983b:186) gehen, wie Büchners Lenz im Meridian auf dem Kopf geht, sich den Atem und das Wort verschlagen lassen, gelangen die Texte an den Punkt der Umkehr, die „Atemwende“ - nur durch das Heraustreten aus bekannten Sinnbezügen kann ein „Anderes“ erfahrbar werden.

13 Celans referenzloses Sprechen hebt PETER SzONDI (1978:348f.) unter Bezug auf Derrida hervor. 
Zur Artikulation von Gefühlen in meineidiger Sprache in einigen Gedichten ...

\section{Gegen das „Heddergemüt“}

Das Misstrauen gegenüber sprachlichen Kodierungen von Emotionen wird in Celans Texten zum Impuls, die angetroffene Sprache von Emotionalität zu reinigen. Seit dem Band Niemandsrose greift der Lyriker auf Fachsprachen zurück und zielt damit auf eine Anreicherung der dichterischen Sprache mit neuen, unverbrauchten Elementen, nachdem die alltagssprachlichen Sprachregister für abgenutzt befunden wurden. Fachsprachliches Vokabular ist weniger emphatisch und mit vorgegebenen Emotionen gesättigt, wurde in der NS-Zeit weniger instrumentalisiert und von den ,tausend Finsternissen todbringender Rede“ (CELAN 1983b:186) vereinnahmt. Vor allem im Spätwerk werden naturwissenschaftliche Termini aus den Bereichen Medizin, Technik oder Neurologie in die Texte einbezogen (vgl. WöGERBAUER 2002:131). Insbesondere die Querverbindungen von Lyrik und Neurophysiologie sind in Celans Gedichten zahlreich. Die Hirnmotivik nimmt in der späten Lyrik zu, Schläfenzange, Nervenzellen, Hirnsichel, Schläfenlappen, Gehörgang, Sehspur, Sehstamm, Herzstamm finden Eingang in die Texte. Die Begriffe werden vom dichterischen Idiom durchdrungen, dabei analysiert, ihrer fixen Bedeutungen entkleidet und in einen Prozess der Re-Semantisierung eingebunden, der als „Akt der Freiheit“ (CELAN 1983b:189) den symbolischen Codierungen der Sprache zuwiderläuft. Aus kleinsten Partikeln soll sich eine neue Sprache wiederaufbauen, in der die Erfahrung des Subjekts aufgehoben ist. Der Prozess der Re-Semantisierung wird in Celans Wortlisten zu Sprachgitter (CELAN 1996:113f.) vorgeführt, die die Textgenese nachvollziehen lassen. Sie beinhalten Worte und ihre Abwandlungen, kreative Ansätze der Variation und Reassoziation. Durch die Koppelung der Elemente wird ihnen neues Potenzial abgewonnen. Es handelt sich bei Celans Wortbildungen häufig um keine lexikalischen, wohl aber semantische Neologismen. Durch die Arbeit am vorgefundenen Sprachmaterial wird ein Durchdringen zu tiefer liegenden Prozessen, zu den Vorstufen des „Denkens“ möglich, denn sie bewegt sich eher in einer psychischen als in einer sprachlich-symbolischen Realität (vgl. MOSER 2005:427). Es werden affektive Prozesse aktiviert, die als Movens des Aufbrechens der symbolischen Ordnung wirksam werden.

Die Arbeit an der Sprache als „Durchlaufen eines Nullpunktes oder semantischen Abgrunds“ (WÖGERBAUER 2002:131) führt der Beginn des Gedichts Seelenblind aus dem Band Fadensonnen von 1967 vor: „Seelenblind, hinter den Aschen,/ im heilig-sinnlosen Wort,/ kommt der Entreimte geschritten,/ den Hirnmantel leicht um die Schultern“ (CELAN 1983a:183). Das „Entreimen“ steht hier stellvertretend für die Negierung ästhetischer Kodierungen 
von Gefühlen: Das dichterische Wort muss durch den Abgrund der Sinnlosigkeit geführt werden. Angesichts der Traumatisierung des Subjekts, auf die das Wort „Seelenblind“ verweist, verzichtet Celan auf die „Fühlwörter“ und nimmt Rekurs auf die Neurologie. Der „Hirnmantel“, ein bestehender neurologischer Fachterminus, wird metaphorisiert und mit neuer Bedeutung angereichert. Der Mantel gewährt Schutz vor traumatisierenden Verletzungen. Damit wird die Schutzfunktion des Hirns gegenüber der Außenwelt benannt, wie sie in Offene Glottis festgeschrieben wird. Angesichts der angetroffenen, meineidigen Sprache und der eigenen traumatischen Versehrtheit tritt der Dichter den Rückzug auf das Neurologische als des einzig Intakten an. Das zerebrale Leitorgan mit seinen neurologischen Funktionen bietet der Subjektivität einen letzten Zufluchtsort. Weitab von sprachlichen Codierungen, bis ins Innerste verletzt und verstört, vergewissert sich das lyrische Ich seiner basalen Fähigkeiten. So erscheint in Offene Glottis ,der Schläfenlappen intakt, wie der Sehstamm“ (CELAN 1983a:388). Der Schläfenlappen oder Temporallappen gehört zum Großhirn, dem phylogenetisch jüngsten Teil des zentralen Nervensystems. Er beherbergt das ,Wernickesche Sprachzentrum', sein Ausfall führt zur sensorischen Aphasie, Wortverständnis und die Kontrolle über die eigene Sprache sind gestört. Der Sehstamm hingegen ist ein Neologismus, Celan kontaminiert die bestehenden Termini „Sehrinde“ oder „Sehsphäre“, die für die Weiterleitung der visuellen Wahrnehmungen verantwortlich sind, mit dem Hirnstamm, dem ältesten Teil des Gehirns, der die grundlegenden und unwillkürlichen Funktionen wie Atmung, Herzschlag und Verdauung reguliert. Celan erkundet das Feld der Neurologie mit seinen eigenen poetischen Ausdrucksmitteln. In seinen Gedichten sind Spuren einer impliziten Neuropoetik auszumachen, das zerebrale Leitorgan wird als zentrales Medium der Aneignung bzw. Herstellung von Welt performativ zur Geltung gebracht. Damit erfährt in seinen Gedichten das sprachliche Ausformung, was in der Neurophysiologie bei der Untersuchung der neurobiologischen Determinanten des Menschen zur Disposition gestellt wird: die Frage nach der Authentizität von Wahrnehmung, vor allem aber der Selbstbestimmtheit des Subjekts. Celan verlegt die Diskussion auf das Feld der Poesie, in seinen Gedichten ist ein fester Subjektstandpunkt nicht besetzbar: „unbesetzbar/ ich und auch du“ (CELAN 1983a:388). Im Gedicht Bon vent, bonne mer heißt es entsprechend: „ein flackernder/ Hirnlappen, ein/ Meerstück,/ hißt, wo du lebst,/ seine Hauptstadt, die/ unbesetzbare“ (CELAN 1983:404). Die Hirnmotivik in Celans Spätwerk ist aber keineswegs rein metaphorisch zu verstehen. Aufenthalte in Pariser psychiatrischen Kliniken verweisen auf konkrete Erfahrungen. Celan war zudem einschlägig gebildet, 
machte sich mit aktuellen psychiatrischen Behandlungsmethoden vertraut. Die Gedichte lassen sich als Reflex auf die Sezierung der Psyche in der psychiatrischen Klinik lesen: ,die Zerstörungen reichen bis in den Kern meiner Existenz ... Man hat mich zerheilt!“ (Zit. nach EMRICH 1999:159f.) Im Nachvollziehen der Vivisektion seines Bewusstseins mit den Mitteln der Psychiatrie betritt der Dichter die letzten Refugien seiner Unversehrtheit: „Heddergemüt, ich kenn// Deine wie Kleinfische wimmelnden/ Messer,// härter als ich/ lag keiner am Wind,// keinem wie mir/ schlug die Hagelbö durch/ das seeklar gemesserte Hirn.“ (CELAN 1983a:225) Noch in dieser äußersten Reduzierung und Verletzung des Ich ist hier ein Subjektstandort bewahrt, von dem aus das „Gemüt“ als ,nicht mehr in einzelne Gefühlswerte zerlegbar[es]“ (FRIEDRICHS 1985:17) Konglomerat von vorfabrizierten Gefühlen mit harten, disharmonischen Worten angegangen wird. Celans Sprache wählt den Weg des sezierenden Analysierens der Gefühlssprache, in der sich das einzelne Subjekt nur noch verfangen, nicht aber aufgehoben wissen kann.

Celans Gedichte erschließen das Niemandsland zwischen Neurologie und Poetik, indem dichterisches Sprechen den neurologischen Funktionen nachgebildet wird. In der Rückbindung an die neuronalen Korrelate emotionaler Vorgänge insistieren Celans Texte auf einer nicht-symbolischen Qualität von Emotionen. Misstrauisch gegenüber symbolisch codierten Emotionen in „todbringender Rede“ (CELAN 1983b:186), tastet sich lyrisches Schreiben an den basalen neurologischen Prozessen voran und baut sich aus diesen wieder auf. Man könnte so weit gehen, Celans - nicht nur neurologische - Neologismen als Nachbildungen neuronaler Verschaltungen zu beschreiben, aus denen sich jenseits von sprachlichen Codierungen ein neues Sprechen entfalten soll. Vielleicht ist damit auch für Celan ,in Neurologie die Poetik der Zukunft versteckt“" (GRÜNBEIN 1996:20), ähnlich wie für den Lyriker Durs Grünbein, der Dichtung als „Vexierbild physiologischen Ursprungs, ähnlich dem Nervensystem“ (GRÜNBEIN 1996:18) beschreibt. Mit Sicherheit aber ist die systematische Etablierung der Querverbindungen von Neurologie und Poetik in Celans Werk ein wichtiges Forschungsdesiderat.

\section{Rudimente einer Sprache der Liebe}

In einem Bertolt Brecht gewidmeten Gedicht wird dessen Diktum, nach den Grauen des Zweiten Weltkriegs sei ein Gespräch über Liebe „beinahe ein Verbrechen“, weil es so viel Ungesagtes einschließe, dahingehend ausgewei- 
tet, dass das Gespräch überhaupt unmöglich geworden sei, „weil es soviel Gesagtes mit einschließt“" (CELAN 1983a:385). Das Gedicht kann somit seine kommunikative Funktion nur wahrnehmen, wenn es am „Gesagten“ vorbeiführt, das symbolische System der Sprache umschifft. Der Weg zum Gegenüber, zum Du des Gedichts ist durch die Sprache, das Medium der Kommunikation verstellt. Damit ist lyrisches Sprechen vom Verstummen bedroht, speist sich aus der Ambivalenz, sich einerseits aus der Sprache zurückziehen und andererseits dem Wunsch nach Ich-Artikulation nachgeben zu wollen. In seinen Gedichten erprobt Celan ein lyrisches Sprechen, das das Schweigen einschließt und symbolische Codierungen unterläuft. Wie ein solches sich in Schweigen hüllendes Sprechen aussehen kann, führt folgendes Gedicht vor: „Seit Langem bestiegener Schlammkahn.// Ein ab-/ Gesprungener/ Knopf/ Tüftelt an jeder Ranunkel,// die Stunde, die Kröte,/ hebt ihre Welt aus den Angeln.// Wenn ich die Karrenspur fräße,/ wär ich dabei.“ (CELAN 1983a:254)

Dem Gedicht sind zeitliche Dimensionierungen eingeschrieben, Vergangenes und Hypothetisches wird in die Gegenwart hineingeholt. Der Vergangenheitsbezug wird durch den adverbialen Einstieg „Seit Langem“ signalisiert. Gegenwart wird im Text jedoch nur als „blinder Fleck“ manifest: Im Zentrum des Gedichts steht ein Ereignis, das sich der genaueren Bestimmung entzieht und allein auf Gegenwärtigkeit beruht. Das lyrische Ich positioniert sich außerhalb, ist nur im Partizip „,bestiegen“ enthalten, verbleibt dennoch in einer dialogischen Bezogenheit auf das Geschehen. Quasi am Rande des Textes stehend, schreibt es auf die Möglichkeit hin, „dabei“ zu sein, die bis zum Schluss im Modus des Hypothetischen bleibt.

Die Schwelle, auf der das sprechende Ich sich situiert, ist die zwischen Lebenden und Toten. Der Text realisiert eine vielfach gebrochene mythologische Ebene der Fahrt ins Totenreich. Es lässt sich eine Analogie zu Kafkas Gracchus-Fragmenten ziehen. ${ }^{14}$ Der vor vielen Jahren im Schwarzwald verunglückte Gracchus ist ein Untoter, der als Barkenfahrer zwischen dem Diesseits und dem Jenseits umherdriftet. Durch ,eine falsche Drehung des Steuers, einen Augenblick der Unaufmerksamkeit des Führers“ hat sein „Todeskahn" die Abgeschiedenheit des Totenreichs verfehlt. Wie das lyrische Ich in Celans Gedicht erwacht Gracchus immer wieder auf seinem ,alten in irgendeinem irdischen Gewässer öde steckenden Kahn“ (KAFKA 1993:309).

14 Zu den Hintergründen von Celans Kafka-Lektüren vgl. LISKA (2006:215-218). Das Gedicht Seit langem wird von Liska nicht erwähnt. 
Wie Gracchus ist das lyrische Ich in Celans Text ein Untoter, oder - um das dramatische Pendant des Gracchus-Komplexes heranzuziehen - ein „Gruftwächter". Es geht sowohl in Kafkas Fragment als auch in Celans Gedicht um die Präsenz von Toten, die die Lebendigen bedrängen. Wie Gracchus gehört auch das lyrische Ich bei Celan nicht mehr ganz zu den Lebendigen, ist unterwegs zwischen dem Totenreich und den Lebenden. Die Toten bleiben, in Form einer Aussparung einer konkreten Referenz, verborgen im Text, womit das Verschweigen, das fehlende Totengedenken, performativ im Text realisiert wird und dem Gedicht die Struktur eines Geheimnisses gibt. Der „Schlamm“ evoziert das Verdrängen der „Leichen im Keller“. ${ }^{15}$ Die Verdrängung des Geschehenen schafft eine spezifische Atmosphäre, in der sich das lyrische Ich in Celans Text bewegt. Am Ende des Textes ist der Schlamm bereits eingetrocknet: der Prozess zunehmender Verhärtung hat eingesetzt. Nur solange der Schlamm noch nicht trocken ist, kann er als lebensspendende Materie wirksam werden, kann sich in ihm neues Leben entfalten.

Das Ich trägt die Signatur der Versehrtheit. Im Gedicht kommt ein traumatisiertes Ich zur Sprache, das unfähig zu Trauer ist. Folgt man Aleida Assmann, ist das Trauma „als eine körperliche Einschreibung ... (zu verstehen), die der Überführung in Sprache und Reflexion unzugänglich ist und deshalb nicht den Status von Erinnerungen gewinnen kann.“ (ASSMANN 1999:278) Der Text bildet die traumatische Fixierung nach, im Zuge deren die Überführung des Erlebten in den Hippocampus nicht gelingt und die traumatische Erinnerung im impliziten Gedächtnis der Amygdala fixiert bleibt. Die traumatische Erinnerung bleibt dort quasi stecken und kann weder zeitlich chronologisch noch verbal erinnert werden. Sie ist fragmentiert, besteht aus einzelnen Erinnerungsbruchstücken, die mittels einschießender Traumabilder oder Flashbacks ins Bewusstsein dringen, allerdings in verschlüsselter Form, so dass sie weder unmittelbar erkannt noch gedeutet werden können. Erneut wird das Gegeneinander von Trauma und der Schutzfunktion des Bewusstseins nachgebildet. Indem das Ich ein unbelebtes Subjekt (den Knopf) aus sich heraustreibt, führt das Gedicht die Reaktion des traumatisierten Subjekts vor, die das Trauma erlebenden Persönlichkeitsanteile abzuspalten, das lyrische Ich wird in ein das Trauma nicht erlebenden emotional unbeteiligten Person und den das Trauma erlebenden hochgradig emotionalen Persönlichkeitsanteilen aufgespalten. Überdies verweist der Knopf als Projektion des

15 Wie präsent die Schlamm-Metapher zur Versinnbildlichung der Atmosphäre der Nachkriegszeit ist, zeigen neuere Texte der Holocaust-Literatur (vgl. RosENBERG 2013:136, 144). 
Beate Sommerfeld

Ich auf dessen Selbstgefühl, mitten im Lebendigen als Toter weiterzuleben, das konstitutiv für die traumatisierte Persönlichkeit ist. Das Ich empfindet sich als toten, unbelebten Gegenstand, womit der Text auf die der Trauer eingeschriebene Ambivalenz verweist: das Schuldgefühl gegenüber den Toten, die Scham, überlebt zu haben, führt zu einer Art Mimikry-Reaktion, einer Anverwandlung an den Tod. ${ }^{16}$

Indem das lyrische Ich solchermaßen aus dem Text herausgehalten wird, wird hier wiederum über das Medium der Kunst „Ich-Ferne“ hergestellt, und der lyrische Text kann als Schutzraum zur Artikulation von Emotionen wirksam werden. Das Ich wird zu einer fluktuierenden Projektion und sprachlichen Leerstelle, der keine feste Position im Symbolischen entspricht, und die im Text beschriebene Welt muss als Emanation des Ich gelesen werden. Das Innere wird im Bild externalisiert: ein Kahn, der im Schlamm unterwegs ist, Kröten, Unkraut sind Rudimente des Subjekts. Die Szenerie gehört zu den verödeten Landstrichen, für die paradigmatisch das Gedicht Fadensonnen (CELAN 1983a:26) steht, der Text bietet sich als „ein Gelände des Todes und des Traumas“ (SzONDI 1978:348) dar. In diesen „Landschaften mit Urnenwesen“ (CELAN 1983a:59) wird eine Trümmerlandschaft gesichtet - auch eine emotionale. Hier wird eine Welt dargestellt, aus der jegliche Emotionalität herausgenommen wurde. Das Gedicht zieht sich in die Sphäre des Biologischen, „Kreatürlichen“ zurück, affekterfüllte Begegnungen werden durch das rein biologische Fortpflanzungsgeschehen substituiert. Das Einarbeiten eines fremden Elements, des Knopfes, führt das Moment des Inkompatiblen ein. Der Knopf zerstört das Naturbild. Nur von weitem verweist er - von der Kleidung ,abgesprungen“ - metonymisch auf das menschliche Subjekt. Sein „Tüfteln“ an der Pflanze verweist auf die instrumentelle Vernunft, die dem naturhaften Geschehen gegenübersteht. Es wird ein absurdes Bild zustande gebracht, womit die Erwartung sinnhafter, quasi natürlicher Rede unterlaufen wird. Die Absurdität des Bildes dient der Affektvermeidung, das Gedicht dient als Schutzraum, in dem Affekte zugleich ausagiert und vermieden werden können. Der Zurücknahme von Emotionalität dienen auch die Abstände zwischen den Zeilengruppen. ${ }^{17}$ In ihnen wird das Geschehen neu organisiert, es werden neue Elemente eingeführt, die mit den vorangegangen durch Kontiguitätsbeziehungen verbunden sind. In dem Pflanzennamen „Ranunkel“ ist

16 In der Trauma-Forschung ist dieses Gefrieren als ,Freeze-Reaktion“ benannt (HUBER 2007:43, 45).

17 MOSER (2005:449) spricht von einer emotionalen Sicherheitsregulierung. 
die Unke verborgen, die wiederum zur „Kröte“ hinüberleitet. Zugleich assoziiert die Unke die unheilverkündenden Unkenrufe. Die Offenbarung der Natur im Sinne selbstevidenter Einheiten wie in der Erlebnislyrik mutiert zur Dechiffrierung arbiträrer Zeichen, die sich nicht in ein Naturbild fügen lassen. Hier ist es Celan jedoch nicht um Referenzlosigkeit im Sinne einer Autonomie des modernen Kunstwerks zu tun (vgl. FRIEDRICHS 1985:143; LAMPING 1989:254), vielmehr darum, ,nicht an die Realität des Todes und der Vernichtungslager zu rühren und so zu tun, als ließe sich ein poetisches Bild von ihnen machen.“ (SzONDI 1978:349) Die Metaphorik der „Lesbarkeit der Natur" bei Goethe und Schiller, wie sie von HANS BLUMENBERG (1986:214232) ins Feld geführt wird, wird zu einer Unlesbarkeit umgedeutet. Deren einziges Signifikat ist der Nachweis einer ungebrochenen Kontinuität der Natur, der die Aufhebung der Signatur der Zeitlichkeit in einen übergeordneten Zusammenhang kaum zu kompensieren vermag. Dem Tod kann nur noch ein zyklisches Modell einer sich immer wieder regenerierenden Natur entgegengehalten werden, das indes nicht zu Bedeutungszusammenhängen codiert wird - etwa im Sinne christlicher Auferstehung. Jenseits der Emotionscodes sucht der Text die Ebene des „Kreatürlichen“ und versucht so, die Balance zwischen Sprechen und Schweigen zu halten. Die Liebe muss das größte Opfer bringen: Sie darf ihren Namen nicht nennen, der Liebescode darf nicht benutzt werden. „Die Liebe löscht ihren Namen: sie/ schreibt sich dir zu.“ (CELAN 1983:225) - so schreibt Celan im Gedicht Zwölf Jahre aus der Niemandsrose. Im Sinne des Satzes des Mystikers Wilhelm von St. Thierry „,amor autem suo defectu plus proficit ${ }^{\star 18}$ fordert Celan die Selbstauslöschung der Liebe, um sie weiter zum Du voranschreiten zu lassen. Nur eine sich selbst auslöschende Liebe als namenlose Anrede des Du kann im Durchstreichen ihres Namens die Bewegung des Gedichts offenhalten. Damit liegt das traditionelle ,Liebesgedicht' in Trümmern vor uns, führt nur noch ein rudimentäres Dasein im Schatten der Geschichte.

Celans Texte signalisieren den Kampf um das Wort und gegen die meineidige Sprache, die nur falsche Emotionen transportieren kann. Am Ausgang des Textes steht das mentale Fixieren eines Problems: Die symbolische Ordnung, in der Gefühle artikuliert werden könnten, ja, die nötig ist, um Emotionen zu artikulieren, ist die gleiche, die in Auschwitz falsifiziert wurde. Es bleibt die Zertrümmerung der Sprache, womit sich das Subjekt zugleich der Möglichkeit der Artikulation von Emotionen beraubt. Zusammenhänge im Sinne des

18 Wilhelm von St. Thierry: De natura et dignitat amoris. Brief 18:80-104 (zit. nach RUH 1993:208). 
Logos werden zerschlagen, es bleiben nur erahnbare assoziative Verknüpfungen bestehen, die im Dunkeln bleiben und auf den Erfahrungshorizont des Ich verweisen. Indem die Wörter die sprachlichen Symbolisierungen durchqueren, übersteigen sie ihre Referenzialität, und der Text verweigert sich dem Anspruch auf Repräsentation von Wirklichkeit (vgl. SzONDI 1978:348), macht sich dafür zum Terrain des Auseinanderschreibens der symbolischen Ordnung - aber auch zum Gelände, das neu beschrieben werden kann. Die Substitution eines Gegenstands durch das Zeichen, das seine Abwesenheit ausfüllen und Präsenz erzeugen soll, wird abgelehnt. Die Spur fungiert als Index, der in ihr figurierte Verweischarakter unterläuft die Logik der Substitution: Die Spur verweist, ohne zu ersetzen, sie bezeichnet, ohne den Gegenstand im Zeichen präsent zu machen und entzieht ihn damit der Symbolisierung. In dieser nicht arretierbaren Verweisstruktur wird die Spur zum textlichen Gegenstück der Trauer, denn wie die Liebe nach dem Sich-Verströmen trachtet, will die Trauer sich verausgaben, hat die Struktur eines endlosen Begehrens nach dem Abwesenden. Die Struktur der Spur entspricht der stets in Bewegung befindlichen Trauer, der Abundanz des Gefühls, das sich verströmen will. Nicht Substitution durch das Bild, sondern die Zeichenpraktiken der Kontiguität und Indexikalität (wie sie in der „Spur“ anklingen) sind Formen der trauernden Verweisung, der es nicht darum geht, die materielle Anwesenheit ihres Gegenstands zu repräsentieren, sondern sie in der Insistenz des Verweisens virulent zu halten (vgl. HORN 1998:239).

Damit wird der Text dynamisiert - das Gedicht bietet sich als ein Vorangehen dar. Indem er nur die Spur gelten lässt, will der Text seine eigene Textualität auflösen, ähnlich wie sich in der Engführung (CELAN 1983:195-204) Grundwasserspuren und Rillen durch den Text ziehen und ihm seine einzig mögliche Konsistenz verleihen. Wird die Spur als autoreferenzieller Verweis auf den Schreibprozess verstanden, wird sie als Kritik an der mit dem Logos, dem Tod, dem Namen gleichzusetzenden Schrift lesbar. Diese soll durch die Spur ersetzt werden, die mit der Stimme, der Selbstpräsenz, dem Atem verbunden wird und eine Sprache konnotiert, die nicht vollständig zum Gerinnen gebracht wurde. Die Spur ist indefinit, erhält das sprachliche Material in der Offenheit der Bewegung, in ihr ist die Sprache auf Widerruf fixiert. Im Evozieren der Spur wird es möglich, den Text gerade in der Bewegung seines Sich-Zurückziehens zu exponieren. Der Spur ist eine Ambiguität eingeschrieben, die Celan dem Schreiben zuordnet, das zugleich die kreatürliche 
Zur Artikulation von Gefühlen in meineidiger Sprache in einigen Gedichten ...

Bewegung des Atmens abbildet ${ }^{19}$ und deren Gerinnen zur Schrift erfordert, eine Ambiguität, die in der Metapher des „Atemkristalls“ figuriert wird. Die Spur bildet zwar Bewegung ab, aber die Bewegung der Spur entsteht als Verbergung ihrer selbst, denn die Spur verstellt auch die Bewegung. Im Wunsch nach dem Tilgen der Spur in neuen Überschreibungen inszeniert das Gedicht die Doppelnatur von Bewahren und Überschreiben, von Sichtbarkeit und Nicht-Zugänglichkeit der Erinnerungsspuren, wie sie im freudschen Wunderblock symbolisiert werden. ${ }^{20}$ Nur in der Schwebe von Bewegung und Fixierung, Auslöschung und Überschreibung erhält lyrisches Sprechen seinen dialogischen Charakter, seine Offenheit, mit der es sich auf ein Anderes zubewegen kann. Celans Spur ist nicht als bildliches Korrelat einer eigenbewegten Sprache, eines „des autonome[n] Bewegungsgefüge[s] der Sprache“ $\mathrm{zu}$ verstehen, als das Friedrichs das moderne Gedicht kennzeichnet (FRIEDRICHS 1985:18). Es wird vielmehr ein Kampf um einen Subjektstandpunkt, um Ich-Präsenz im Text gefochten. Träumt das Ich am Anfang noch aus einer textlichen Randposition heraus davon, „dabei“ zu sein beim Textgeschehen, imaginiert es zuletzt mit dem „Fressen“ der Karrenspur einen fast schon aggressiven Zugriff auf die Eigenbewegung der Spur, um als sprechendes Subjekt im Text zu Wort zu kommen.

19 Vgl.: „Das Gedicht ... es kommt immer noch auf Atemwegen“ (CELAN 1999:51). Celan notierte sich in den Entwürfen zum Meridian: „,Was auf der Lunge, das auf der Zunge “ - pflegte meine Mutter zu sagen - auf Atemwegen kommt es, das Gedicht, pneumatisch ist es da: für jeden." (Celan 1999:51); vgl. ebenfalls zur Erläuterung bei BösCHENSTEIN (2000:645). Celans Texte sollen so weit wie möglich dem Atmen nachgebildet werden. Wie konstitutiv für Celan das Atmen für die dichterische Arbeit ist, zeigt seine Bitte bezüglich der Herausgabe seiner Rede, den Text ,unter Berücksichtigung aller Leerzeilen abzudrucken, denn: „diese Leerzeilen sind nämlich keineswegs typographische ,Streckungen'; sie gehören vielmehr zum Text selbst; sie heben die Atem- und Bedeutungseinheiten hervor" (CELAN 1999:254). In der poetologischen Festschreibung der Verbindung der kreatürlichen Bewegung des Atmens und dichterischem Sprechen greift Celan eines der ältesten Topoi überhaupt auf. Der Phonozentrismus Rousseaus denunziert die fehlende Selbstpräsenz der Schrift im geläufigen Sinne, die er als toten Buchstaben kennzeichnet, der dem Leben den Atem benimmt. Auf der anderen Seite wird die Schrift im metaphorischen Sinne, die natürliche, göttliche und lebendige Schrift verehrt, sie ist unmittelbar an die Stimme und den Atem gebunden (vgl. DERRIDA 1974:33).

20 Vgl. JAGOW / STEGER 2004:51-65, S. 57; MEYer (2002:112-130); zur Quelle: FREUD (1925:1-5); vgl. ebenfalls ASSMANN (1999:151-158, insbesondere 156). 
Beate Sommerfeld

\section{Schlussbemerkungen}

Celans Gedichte stellen sich den Gattungserwartungen, indem sie die Möglichkeiten der Artikulation von Emotionen im Gedicht erkunden. Im Abarbeiten an den textlichen Codierungen von Emotionen erweist sich, dass Literatur imstande ist, das kulturelle Ausdrucksarsenal an Emotionen um Variationen und Differenzierungen von Gefühlen zu erweitern. Das Gedicht gibt sich nicht dazu her, dem Gemütvollen zum Ausdruck zu verhelfen, das sich nicht in einzelne Gefühlswerte zerlegen lässt (FRIEDRICHS 1985:17). Die Gedichte arbeiten den Konzeptualisierungen distinkter Emotionen wie Liebe und Trauer entgegen, da diese bereits in meineidiger Sprache aufgehoben sind. Celan verbleibt jenseits der Emotionscodes, Gefühle sind nur als ein Anderes verfügbar. Die Emotionen, die in Celans Texten zu Wort kommen, entfalten sich an der Schnittstelle von sprachlicher Repräsentation und Affekt und können nur als Selbstreflexion in die Texte eingehen. Letztendlich stehen dabei die Tragfähigkeit von Zeichenordnungen und der Ort des fühlenden und sprechenden Subjekts auf dem Prüfstein. Lyrisches Sprechen bewegt sich damit auf eine Grenze zu, an welcher sprachliche Figurationen, ja Sprache überhaupt fragwürdig werden.

Die Polarität von Modernität und Emotionalität lässt sich im Hinblick auf Celans Texte nicht halten. Noch im selbst aufgezwungenen ,kühlen Operieren“ (FRIEDRICHS 1985:29) mit der Sprache stehen die Gedichte stets unter dem Neigungswinkel eines Ich, das „dabei“ sein will im Textgeschehen. In einer steten reflexiven Rückwendung auf sich selbst und sein Medium, die Sprache, zutiefst verletzt und traumatisiert, setzt sich das sprechende Subjekt im Gedicht neuen Risiken der Versehrung aus, indem es sich auf den Weg zum Anderen begibt. „La poésie ne s'impose plus, elle s'expose“ (CELAN 1983b:181), diese Erkenntnis hat Celan 1969 am Ende seines Lebens als Dichter gewonnen: Das Gedicht setzt sich aus und riskiert eine Öffnung auch für neue Verletzungen und neuen Schmerz. Im Drängen auf Alteritätserfahrungen bleibt das Gedicht „Ein Wurfholz auf Atemwegen“ (CELAN 1983:258). Im Offenhalten der Schrift in kreatürlichem Schreiben, zum Atmen, der Stimme hin, sollen traumatische Verhärtungen gelöst und eine Trauerarbeit initiiert werden, die das Ich auf den Weg schickt zur Begegnung mit dem Anderen: So wird im Gedicht Stimmen dem Atmen zugemutet, die „Tränen im Bruderaug“ (CELAN 1983:147) zu lösen. Die Metapher des Atems ist das treffendste Bild sowohl für Trauer und Liebe als auch für Dichtung, denn der Atem ist Träger der verbindenden Rede vom Ich zum Du und 
hebt die Grenze zum Anderen auf: ,gemeinsam/ laß uns atmen den Schleier,/ der uns voreinander verbirgt“" (CELAN 1983:95).

\section{Literatur}

AnZ, Thомаs (2006): Emotional Turn? Beobachtungen zur Gefühlsforschung: literaturkritik.de 9/12 (23.12.2013).

- (2011): Todesszenarien - Literarische Techniken zur Evokation von Angst, Trauer und anderen Gefühlen. In: EBERT, LISANNE et al. (eds.): Emotionale Grenzgänge. Konzeptualisierungen von Liebe, Trauer und Angst in Sprache und Literatur. Würzburg, 113-129.

AssmanN, AlEIDA (1999): Erinnerungsräume, Formen und Wandlungen des kulturellen Gedächtnisses. München.

BARTHES, RolAnd (1977): Fragments d'un discours amoureux. Paris.

Blumenberg, HANs (1986): Die Lesbarkeit der Welt. Frankfurt (M.).

Böhme, Hartmut (1997): Gefühl. In: Wulf, Christoph (ed.): Vom Menschen. Handbuch Historische Anthropologie. Weinheim/Basel, 525-548.

Böning, Thomas (2005): „Was auf der Lunge, das auf der Zunge“. Paul Celans Offene Glottis - Trauma/Utopie. In: BoRGARDS, RolAND (ed.): Schmerz und Erinnerung. München, 217-244.

BÖSCHENSTEIn, BERnHARD (1988): Celan und Mandelstamm. Beobachtungen zu ihrem Verhältnis. In: Celan-Jahrbuch 2:155-168.

BÖSCHENSTEIN, BERNHARD (2000): Quelques observations sur les choix et utilisations des citations dans les matériaux du «Meridian». In: Etudes Germaniques 55/3: 633-645.

Celan, Paul (1983): Gesammelte Werke in fünf Bänden. Gedichte I. Hrsg. von Beda Allemann und Stefan Reichert unter Mitwirkung von Rolf Bücher. Frankfurt (M.).

- (1983a): Gesammelte Werke in fünf Bänden. Gedichte II. Hrsg. von Beda Allemann und Stefan Reichert unter Mitwirkung von Rolf Bücher. Frankfurt (M.).

- (1983b): Gesammelte Werke in fünf Bänden. Gedichte III. Prosa. Reden. Hrsg. von Beda Allemann und Stefan Reichert unter Mitwirkung von Rolf Bücher. Frankfurt (M.).

- (1994): Schneepart. Hrsg. von Rolf Bücher. (Historisch-kritische Ausgabe I. Abt.). Bd. 10,2 (Apparatband). Frankfurt (M.).

- (1996): Sprachgitter. Vorstufen, Textgenese, Endfassung. Hrsg. von Jürgen Wertheimer. Frankfurt (M.).

- (1999): Der Meridian. Endfassung - Entwürfe - Materialien. Hrsg. von Bernhard Böschenstein und Heinz Schmull. Frankfurt (M.).

Celan, Paul - Celan-Lestrange, Gisèle (2001): Briefwechsel. Mit einer Auswahl von Briefen Paul Celans an seinen Sohn Eric. Hrsg. und kommentiert von Bertrand Badiou in Verbindung mit Eric Celan. 2 Bde. Bd. 2: Kommentar. Frankfurt (M.). 
Beate Sommerfeld

DeRridA, JACQUeS (1974): Grammatologie. Aus dem Französischen von Hans-Jörg Rheinberger und Hanns Zischler. Frankfurt (M.).

- (1988): Mnemosyne. Aus dem Französischen von Hans-Dieter Gondeck. In: Mémoires. Für Paul de Man. Wien, 13-66.

EMrich, Wolfgang (1999): Paul Celan. Berlin.

Freud, Sigmund (1925): „Notiz über den Wunderblock“. In: Internationale Zeitschrift für Psychoanalyse 11/1:1-5.

Frey, Eleonore (1987): La poésie ne s'impose plus, elle s'expose. Zu Paul Celans Poetik. In: Jahrbuch Internationale Germanistik 20:20-36.

Friedrichs, Hugo (1985): Die Struktur der modernen Lyrik. Von der Mitte des neunzehnten bis zur Mitte des zwanzigsten Jahrhunderts. Reinbek bei Hamburg.

FRIES, NORBERT (2007): Die Kodierung von Emotionen in Texten. Teil 1: Grundlagen. In: JLT 1.2:293-337.

GrÜNBEIN, DuRs (1996): Galilei vermißt Dantes Hölle und bleibt an den Maßen hängen. Aufsätze 1989-1995. Frankfurt (M.).

Hegel, Georg Wilhelm Friedrich (1970): Vorlesungen über die Ästhetik III. Theorie. Werkausgabe. Hrsg. von Eva Moldenhauer und Karl Markus Michel. Bd. XV. Frankfurt (M.).

Horn, Eva (1998): Trauer schreiben. Die Toten im Text der Goethezeit. München. Huber, Michaela (2007): Trauma und die Folgen. Bd. 1. Paderborn.

ISER, Wolfgang (1991): Das Fiktive und das Imaginäre. Perspektiven literarischer Anthropologie. Frankfurt (M.).

JAGOW, BetTINA / STEGER, FloRIAN (2004): Bilder des Menschen zwischen Selbstbestimmung und Fremdsteuerung. Ulrike Draesners autopilot-Gedichte. In: JAGOW, BetTina / Steger, Florian (eds.): Repräsentationen. Medizin und Ethik in Literatur und Kunst der Moderne. Heidelberg, 51-65.

KAFKA, FrANZ (1993): Nachgelassene Schriften und Fragmente. Hrsg. von Malcolm Pasley. Frankfurt (M.).

KIESEl, Helmut (2004): Geschichte der literarischen Moderne. München.

КосH, ElKe (2007): Trauer und Identität. Inszenierungen von Emotionen in der deutschen Literatur des Mittelalters. Berlin.

KORTE, HERMANN (1989): Geschichte der deutschen Lyrik seit 1945. Stuttgart.

LACAN, JACQUES (1978): Seminar I. Die vier Grundbegriffe der Psychoanalyse. Aus dem Französischen von Norbert Haas. Olten/Freiburg.

LAMPING, DieTER (1989): Das lyrische Gedicht. Definitionen zu Theorie und Geschichte der Gattung. Göttingen.

- (1991): Moderne Lyrik. Eine Einführung. Göttingen.

LinK, JÜRGEN (1992): Elemente der Lyrik. In: BrackeRT, HeLmut / STRÜCKRATH, JöRN (eds.): Literaturwissenschaft. Ein Grundkurs. Reinbek bei Hamburg, 86-101. 
Liska, Vivian (2006): Ein Meridian wider die Zeit. Von Celan zu Kafka. In: Engel, MANFRED / LAMPING, DieTER (eds.): Franz Kafka und die Weltliteratur. Göttingen, 210-233.

LuHMANN, Niklas (1982): Liebe als Passion. Zur Codierung von Intimität. Frankfurt (M.). Meyer, Anne-Rose (2002): Physiologie und Poesie. Zu Körperdarstellungen in der Lyrik von Ulrike Draesner, Durs Grünbein und Thomas Kling. In: Gegenwartsliteratur 1:107-133.

MEYER-SICKENDIEK, BURKHARD (2011): „Spürest du kaum einen Hauch“. Über die Leiblichkeit in der Lyrik. In: ANDERMANn, KERSTIN / EBERLEIn, UNDINE (eds.): Gefühle als Atmosphäre: Neue Phänomenologie und philosophische Emotionstheorie. Berlin, 213-232.

Moser, Ulrich (2005): Psychische Mikrowelten. Neuere Aufsätze. Hrsg. von Marianne Leuzinger-Bohleber und Ilka von Zeppelin. Göttingen.

NAJDITSCH, LARISSA (1998): Paul Celans Gedicht „Eine Gauner- und Ganovenweise“ im Blick auf die Frage „Celan - Mandelstam“. In: Etudes Germaniques 53: 687-700.

RiEdel, MANFRed (1989): Hören auf die Sprache. Die akroamatische Dimension der Hermeneutik. Frankfurt (M.).

Rosenberg, Göran (2013): Ein kurzer Aufenthalt. Aus dem Schwedischen von Jörg Scherzer. Berlin.

RUH, KURT (1993): Geschichte der abendländischen Mystik. Bd. 2. München.

SCHILLER, FrIEDRICH (1943): Werke. Nationalausgabe. Bd. 1: Gedichte. Hrsg. von Julius Petersen und Friedrich Beißner. Weimar.

SCHUlZ, GEORG-Michael (2002): Eine Gauner- und Ganovenweise von Paul Celan. In: SPeier, Hans-Michael (ed.): Gedichte von Paul Celan. Stuttgart, 105-120.

SZONDI, PETER (1978): Durch die Enge. Versuch über die Verständlichkeit des modernen Gedichts. Von den Herausgebern aus dem Französischen übersetzt. In: SzONDI, PETER: Schriften II. Hrsg. von Jean Bollack et al. Frankfurt (M.), 345-389.

VESTER, HEINZ-GÜNTHER (1991): Emotion, Gesellschaft und Kultur. Grundzüge einer soziologischen Theorie der Emotionen. Opladen.

WeIgel, Sigrid / BÖsCHENSTEIN, BERNHARD (1997): Paul Celan - Ingeborg Bachmann: Zur Rekonstruktion einer Konstellation. In: BÖSCHENSTEIN, BERNHARD / WEIGEL, Sigrid (eds.): Ingeborg Bachmann und Paul Celan. Poetische Korrespondenzen. Frankfurt (M.), 7-14.

Winko, Simone (2003): Kodierte Gefühle. Zu einer Poetik der Emotionen in lyrischen und poetologischen Texten um 1900. Berlin.

WöGerbauer, Werner (2002): Fadensonnen. In: SPeier, HANS-Michael (ed.): Gedichte von Paul Celan. Stuttgart, 121-132. 